\title{
Divergent effects of muscarinic receptor subtype gene ablation on murine colon tumorigenesis reveals association of M3R and zinc finger protein 277 expression in colon neoplasia
}

Kunrong Cheng ${ }^{1}$, Guofeng Xie ${ }^{1}$, Sandeep Khurana ${ }^{2}$, Jonathon Heath ${ }^{3}$, Cinthia B Drachenberg ${ }^{3}$, Jennifer Timmons ${ }^{4}$, Nirish Shah ${ }^{1}$ and Jean-Pierre Raufman ${ }^{1,5^{*}}$

\begin{abstract}
Background: M3 and M1 subtype muscarinic receptors are co-expressed in normal and neoplastic intestinal epithelial cells. In mice, ablating Chrm3, the gene encoding M3R, robustly attenuates intestinal tumor formation. Here we investigated the effects of Chrm 1 gene ablation, alone and in combination with Chrm3 ablation.

Methods: We used wild-type, Chrm1 $1^{-1}$, Chrm $3^{-/}$and combined Chrm $1^{-1 /} / \mathrm{Chrm}^{-1-}$ knockout (dual knockout) mice. Animals were treated with azoxymethane, an intestine-selective carcinogen. After 20 weeks, colon tumors were counted and analyzed histologically and by immunohistochemical staining. Tumor gene expression was analyzed using microarray and results validated by RT-PCR. Key findings were extended by analyzing gene and protein expression in human colon cancers and adjacent normal colon tissue.

Results: Azoxymethane-treated Chrm3 ${ }^{-1-}$ mice had fewer and smaller colon tumors than wild-type mice. Reductions in colon tumor number and size were not observed in $\mathrm{Chrm}^{-1-}$ or dual knockout mice. To gain genetic insight into these divergent phenotypes we used an unbiased microarray approach to compare gene expression in tumors from $\mathrm{Chrm}^{-1}$ to those in wild-type mice. We detected altered expression of 430 genes, validated by quantitative RT-PCR for the top 14 up- and 14 down-regulated genes. Comparing expression of this 28-gene subset in tumors from wild-type, Chrm $3^{-1}$, $\mathrm{Chrm}^{-1-}$ and dual knockout mice revealed significantly reduced expression of Zfp277, encoding zinc finger protein 277, in tissue from M3R-deficient and dual knockout mice, and parallel changes in Zfp277 protein expression. Notably, mRNA and protein for ZNF277, the human analogue of Zfp277, were increased in human colon cancer compared to adjacent normal colon, along with parallel changes in expression of M3R.

Conclusions: Our results identify a novel candidate mouse gene, Zfp277, whose expression pattern is compatible with a role in mediating divergent effects of Chrm3 and Chrm 1 gene ablation on murine intestinal neoplasia. The biological importance of this observation is strengthened by finding increased expression of ZNF277 in human colon cancer with a parallel increase in M3R expression. The role of zinc finger protein 277 in colon cancer and its relationship to M3R expression and activation are worthy of further investigation.
\end{abstract}

Keywords: Muscarinic receptors, M3R, M1R, Chrm1, Chrm3, Mouse models, Colon cancer, Azoxymethane, ZNF277

\footnotetext{
* Correspondence: jraufman@medicine.umaryland.edu

'Department of Medicine, Division of Gastroenterology \& Hepatology and

Program in Oncology, Marlene and Stewart Greenebaum Cancer Center, VA

Maryland Health Care System and University of Maryland School of Medicine,

Baltimore, MD 21201-1595, USA

${ }^{5}$ Division of Gastroenterology \& Hepatology, 22 S. Greene St., N3W62,

Baltimore, MD 21201-1595, USA

Full list of author information is available at the end of the article
}

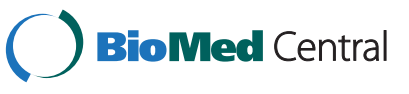

(c) 2014 Cheng et al.; licensee BioMed Central Ltd. This is an Open Access article distributed under the terms of the Creative Commons Attribution License (http://creativecommons.org/licenses/by/2.0), which permits unrestricted use, distribution, and reproduction in any medium, provided the original work is properly credited. The Creative Commons Public Domain Dedication waiver (http://creativecommons.org/publicdomain/zero/1.0/) applies to the data made available in this article, unless otherwise stated. 


\section{Background}

Activation of muscarinic receptors and downstream signaling was shown to stimulate proliferation of cells derived from lung [1,2], breast [3,4], prostate [5], colon $[6,7]$, and skin [8] cancers, and muscarinic receptors are frequently over-expressed in these common cancers [9]. Hence, it is highly likely that activation of muscarinic receptor signaling plays a fundamentally important role in neoplastic transformation and progression.

Of five cholinergic muscarinic receptor subtypes, designated M1R - M5R [10], human colon cancer cells express primarily M3R. M3R activation stimulates cell proliferation, survival, migration and invasion [6,11-14] - key hallmarks of neoplasia [15]. Human colon cancer cells also produce and release acetylcholine at concentrations capable of activating M3R and stimulating cell proliferation, identifying the capacity for autocrine and paracrine stimulation of M3R signaling [11]. Jointly, these in vitro studies provided strong evidence that M3R expression and signaling are particularly important in the progression of colon neoplasia.

Mice with targeted knockout of genes encoding each of the five muscarinic receptor subtypes (Chrm1 - Chrm5) are useful for investigating their biological functions [16-18]. We showed that ablating Chrm3, the gene encoding M3R, attenuates colon neoplasia in mice treated with azoxymethane (AOM), a colon-selective carcinogen [19]. Compared to AOM-treated WT mice, AOM-treated Chrm3 knockout mice had $40 \%$ and $60 \%$ reductions in tumor number and size, respectively. Similar results were obtained using $A p c^{\mathrm{min} /+}$ mice, a genetic model of intestinal neoplasia [20]. These findings suggested to us that treatments directed at reducing M3R expression, activation or downstream signaling might be useful to prevent or treat colon neoplasia. Indeed, $A p c^{\text {min/+ }}$ mice treated with scopolamine butylbromide, an inhibitor of muscarinic receptor activation, developed fewer intestinal tumors than vehicle-treated control mice [20].

Many cell types co-express muscarinic receptor subtypes [17]. Using in situ hybridization, we demonstrated expression of mRNA for both M1R and M3R in murine gastric [21] and colonic [19] epithelial cells. Likewise, human colon cancer cells used to investigate in vitro actions of muscarinic receptors and ligands express a mixture of M3R and M1R, with a predominance of M3R [13,22]. Whereas expression of multiple muscarinic receptor subtypes in the same cell type is likely to provide growth and survival advantages, it can also result in complex, unpredictable interactions. This was apparent when we examined the impact of Chrm1 and Chrm3 co-expression in gastric chief cells that synthesize and release the proenzyme pepsinogen [21]. M3R deficiency did not alter carbamylcholine (carbachol)-induced pepsinogen release, but M1R deficiency resulted in a $25 \%$ decrease in pro- enzyme release [21]. Strikingly, in mice deficient in both M1R and M3R, carbachol-induced pepsinogen secretion was totally abolished [21].

These observations motivated us to examine the role of M1R (Chrm1) expression in colon neoplasia and to determine whether knocking out both M1R (Chrm1) and M3R (Chrm3) in the same animal (hereafter called dual $\mathrm{KO}$ mice) would more effectively attenuate AOMinduced colon neoplasia than M3R (Chrm3) knockout alone. We also took advantage of these murine models to identify genes whose expression levels might be relevant to resulting colon tumor phenotypes.

\section{Results}

\section{Effects of M1R, M3R and dual knockout on murine colon} neoplasia

We treated WT, $\mathrm{Chrm3}^{-/-}, \mathrm{Chrm}^{-/-}$and dual $\mathrm{KO}$ mice with phosphate buffered saline (vehicle control) or AOM, a colon-selective carcinogen [19] (Figure 1A). As reported previously [23], at baseline $\mathrm{Chrm}^{-/-}$mice weighed $15-20 \%$ less than WT mice (Figure 1B). At baseline, $\mathrm{Chrm}^{-1-}$ mice also weighed less than WT mice, but in contrast to $\mathrm{Chrm}^{-/-}$mice, their weights approached those of WT mice by the end of the 20-week study period (Figure 1B).

As a group dual $\mathrm{KO}$ mice were extremely frail, necessitating a modified study design; whereas AOM treatment was started in six-week-old $\mathrm{Chrm}^{-/-}$and $\mathrm{Chrm1}^{-/-}$mice, AOM treatment was delayed in dual $\mathrm{KO}$ mice until they were 11 to 12 -weeks-old and better able to tolerate AOM treatment [24]. Even with this modification, at 11-12 of age dual $\mathrm{KO}$ mice weighed $\sim 25 \%$ less than WT mice (Figure $1 \mathrm{~B}$ ), and in contrast to the other genotypes dual $\mathrm{KO}$ mice lost weight during the first six weeks of AOM treatment; at 20 weeks they still weighed $\sim 30 \%$ less than WT mice (Figure 1B).

Although the gross anatomical appearance of colons in knockout mice was normal, we detected a modest but statistically significant reduction in colon length (Figure 1C). These differences probably reflect the lower body weights of $\mathrm{Chrm3}^{-/}$, Chrm1 $\mathrm{CH}^{-/}$and dual $\mathrm{KO}$ compared to WT mice (Figure 1B). Colon length was not significantly different when compared within the three groups of knockout mice. Microscopic review of H\&E-stained tissue sections from untreated (no AOM or vehicle) mice with the four muscarinic receptor genotypes by a senior gastrointestinal pathologist $(\mathrm{CD})$ revealed no differences in colon epithelial morphology (Figure 1D).

Mice treated with vehicle alone did not develop colon neoplasia (not shown). Representative photographs in Figure 2A show robust colon tumor formation in AOMtreated mice. At the 20-week end-point, only colons from $\mathrm{Chrm}^{-/-}$mice had reduced tumor burden compared to WT mice (Figure 2A). Tumor measurements revealed 28 and $74 \%$ reduction, respectively, in tumor number and 

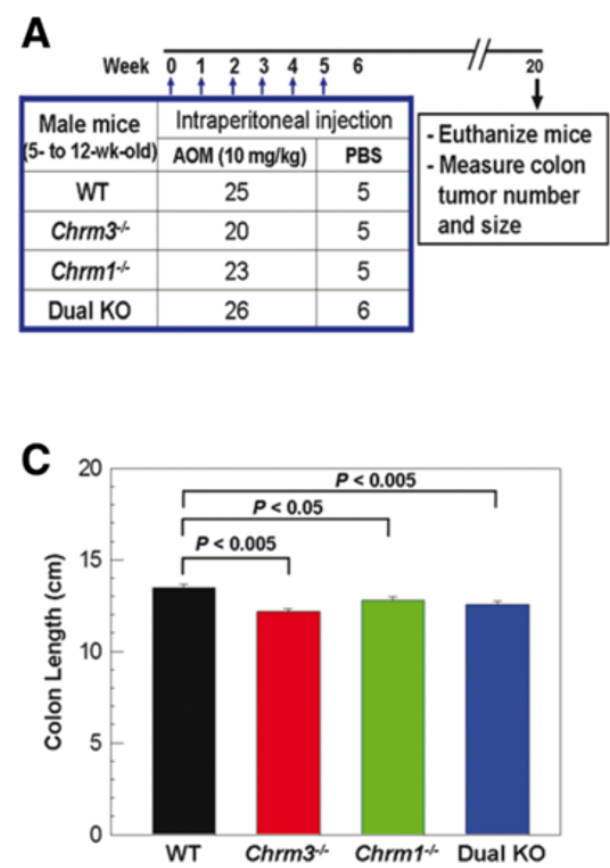

D
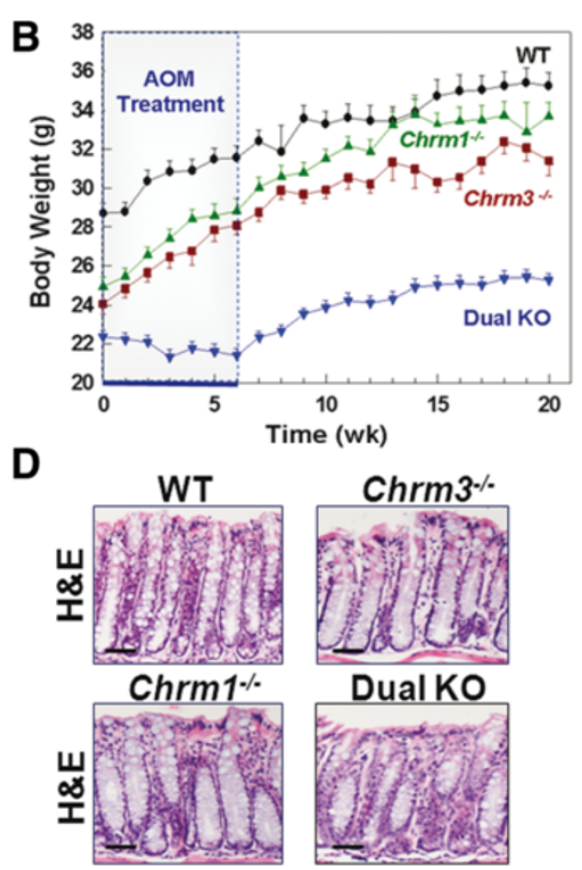

Figure 1 Study protocol, animal weights, colon length and histological appearance of colon sections from mice with different Chrm genotypes. A: Schematic of study design; WT, Chrm $3^{--}, \mathrm{Chrm}^{-1-}$ and dual $\mathrm{KO}$ male mice were treated with intraperitoneal injection of AOM $(10 \mathrm{mg} / \mathrm{kg}$ ) or an equal volume of vehicle (phosphate buffered saline) weekly for 6 weeks and followed for a total of 20 weeks. At 20 weeks, animals were euthanized and colon tumor number and size, and mucosal markers of proliferation and apoptosis were measured. B: Weights of AOM-treated mice during the 20-week study (mean \pm S.E.). C: Colon length of AOM-treated mice was measured following euthanasia at 20 weeks (mean \pm S.E.). D No morphological differences were seen in hematoxylin and eosin (H\&E)-stained microscopic sections of normal colon tissue from WT, Chrm3 ${ }^{-1}, \mathrm{Chrml}^{-1}$ and dual KO mice. Size bars $=50$ micrometers.

size for $\mathrm{Chrm}^{-/}$compared to WT mice $(P<0.05$ and $P<0.005$, respectively) (Figure $2 \mathrm{~B}$ and $\mathrm{C}$ ), consistent with our previous work [19]. When colon tumors were stratified by volume (Figure 2D), a shift in tumor size in $\mathrm{Chrm}^{-/-}$mice towards smaller lesions $\left(<2 \mathrm{~mm}^{3}\right)$ became evident.

We were surprised to observe that genetic ablation of Chrm1 did not alter the number or volume of colon tumors in $\mathrm{Chrm1}^{-1}$ mice compared to WT mice (Figure 2A-D). Even more surprising were the outcomes in dual $\mathrm{KO}$ mice; tumor number and volume were only slightly reduced compared to those in WT and $\mathrm{Chrm1}^{-1-}$ mice (Figure 2A-D). That is, concomitant genetic ablation of Chrm1 in $\mathrm{Chrm}^{-/-}$mice appeared to mitigate reductions in both colon tumor number and volume that we repeatedly observed in AOM-treated $\mathrm{Chrm}^{-/-}$ compared to WT mice (Figure 2A-D) [19].

As shown in Figure 2E, in WT, Chrm1 ${ }^{-/-}$and dual KO mice the majority of colon tumors were adenocarcinomas. In contrast, $\mathrm{Chrm}^{-1-}$ mice had nearly equivalent numbers of adenomas and adenocarcinomas. Although adenomas were numerically less frequent in dual $\mathrm{KO}$ compared to WT mice (Figure 2E), this was not a significant difference $(P=0.1)$. Likewise, there were no significant differences in the numbers of adenocarcinomas per section when comparing $\mathrm{Chrm}^{-1 /}$, dual $\mathrm{KO}$ and WT mice. Conversely, the $76 \%$ reduction in adenocarcinomas in colons from $\mathrm{Chrm}^{-1-}$ compared to WT mice was highly significant $(P<0.001)$ (Figure 2E). These findings suggest that the major impact of M3R deficiency in AOM-treated mice is to block progression of colon adenomas to adenocarcinomas.

We evaluated the multiplicity of adenocarcinomas per section; 56\% of $\mathrm{Chrm}^{-/}$mouse colons had no adenocarcinomas and only one $\mathrm{Chrm}^{-/}$mouse had more than one colon adenocarcinoma. In contrast, more than $50 \%$ of WT, Chrm1 ${ }^{-1-}$ and dual KO mice had multiple (two to seven) adenocarcinomas per section and only two of 20 WT (10\%) and one of 12 dual KO (8\%) mice had no adenocarcinomas $(P<0.01$ for reduced multiplicity of tumors in colons from $\mathrm{Chrm}^{-/}$mice vs. colons from the other three genotypes). Colons from all $\mathrm{Chrm}^{-1-}$ mice contained at least one adenocarcinoma.

We considered the possibility that modifying our protocol to delay AOM treatment of frail dual $\mathrm{KO}$ mice until they were 12 weeks old might have impacted outcomes - AOM treatment in the three other genotypes started when mice were six weeks old. To exclude this as a confounder, we started AOM treatment in WT mice at six $(\mathrm{N}=25)$ or $12(\mathrm{~N}=16)$ weeks of age. Twenty weeks 


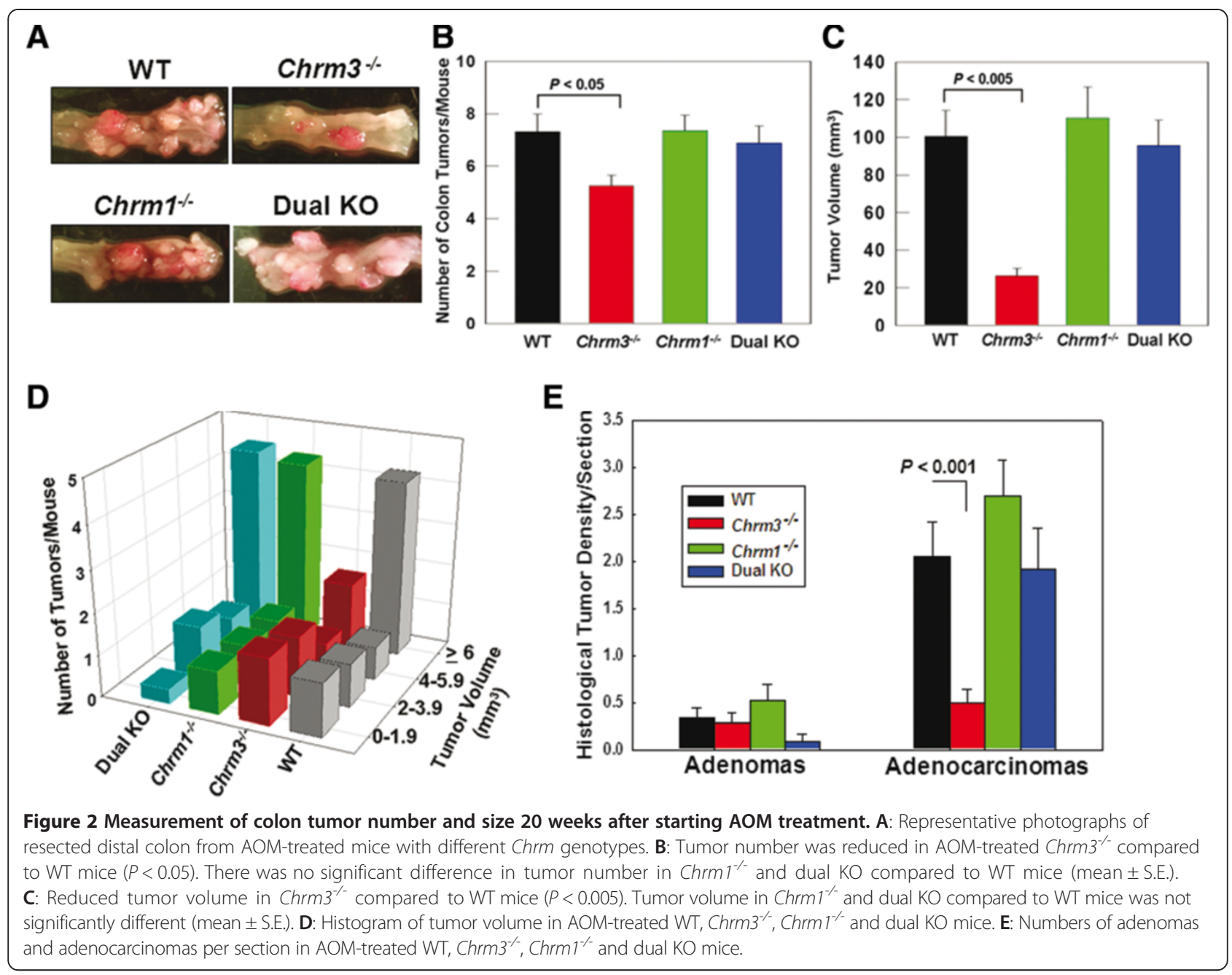

after starting AOM treatment there was no difference in tumor number or volume when comparing mice that started AOM treatment at age six versus 12 weeks (Additional file 1). We concluded that the failure to observe reduced tumor number and size in AOM-treated dual $\mathrm{KO}$ mice cannot be attributed to the delay in initiating AOM treatment.

\section{Effect of M1R, M3R and dual knockout on tumor cell proliferation and apoptosis}

To determine whether changes in tumor number and size resulted from differences in cell proliferation and apoptosis, we examined Ki67 and activated caspase-3 staining, respectively. Figure $3 \mathrm{~A}$ shows representative micrographs of Ki67 staining in adenomas from AOMtreated WT, $\mathrm{Chrm3}^{-/-}, \mathrm{Chrm}^{-1-}$ and dual KO mice. Compared to adenomas from AOM-treated WT mice, Ki67 staining was significantly reduced in those from $\mathrm{Chrm3}^{-/-}$and dual $\mathrm{KO}$ but not $\mathrm{Chrm1}^{-/-}$mice (Figure 3). Figure 3B shows 58\% reduction in Ki67-positive cells in adenomas from $\mathrm{Chrm}^{-/-}$compared to those from WT mice $(P<0.01)$. Ki67 staining was reduced $42 \%$ in dual KO mice $(P<0.05$ compared to WT mice). Thus, although Ki67 staining was significantly reduced in dual $\mathrm{KO}$ compared to WT mice, this reduction was less than that observed in $\mathrm{Chrm}^{-/-}$mice (Figure 3B), suggesting that in AOM-treated mice concomitant ablation of Chrm1 mitigates anti-proliferative effects of Chrm3 gene ablation.

Figure 3C shows representative micrographs of activated caspase-3 staining in adenomas from AOMtreated WT, $\mathrm{Chrm3}^{-/}$, Chrm1 ${ }^{-/}$and dual KO mice. The number of apoptotic cells in adenomas from AOMtreated mice was almost two orders of magnitude lower than the number of proliferating cells (compare scales for vertical axes in Figure $3 \mathrm{~B}$ and D). Apoptotic cells were reduced in adenomas from $\mathrm{Chrm}^{-/}$and dual $\mathrm{KO}$ compared to WT mice $(P<0.01$ and $<0.001$, respectively). In contrast, apoptotic cells were not significantly different in adenomas from Chrm1 ${ }^{-/-}$and WT mice (Figure 3D). 

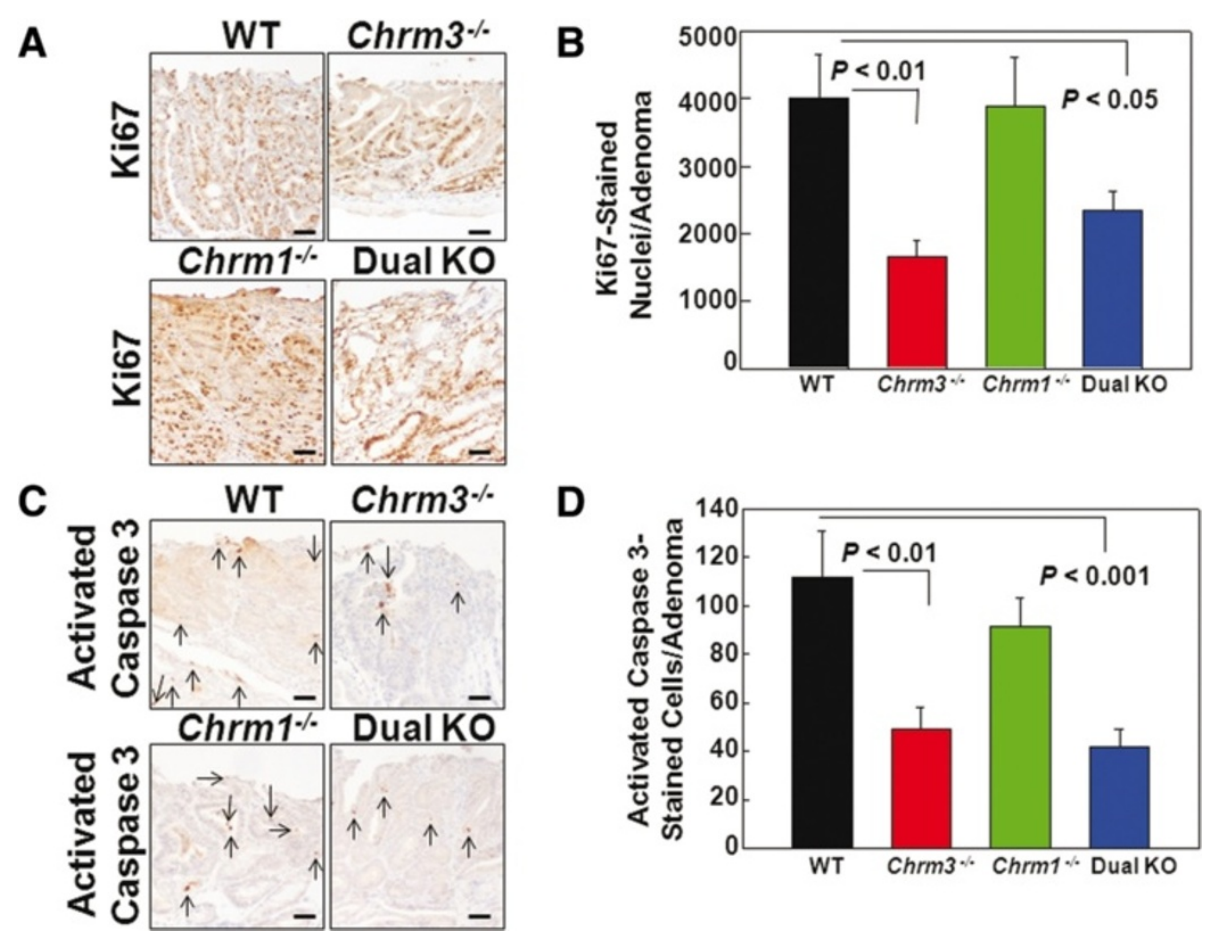

Figure 3 Markers of cell proliferation and apoptosis in colon adenomas from AOM-treated mice. A. Representative staining for Ki67 in adenomas from WT, Chrm3 $3^{--}, \mathrm{Chrm}^{-1}$ and dual $\mathrm{KO}$ mice. B. Ki67 staining was significantly reduced in adenomas from Chrm $3^{-/-}$and dual $\mathrm{KO}$ compared to WT mice. C. Representative staining for activated caspase-3 in adenomas from WT, Chrm3 ${ }^{-\%}$, Chrm ${ }^{-1}$ and dual KO mice. Stained cells are indicated by arrows. D. Activated caspase-3 staining was significantly reduced in adenomas from $\mathrm{Chrm}^{-1-}$ and dual $\mathrm{KO}$ compared to WT mice. For $B$ and $\mathrm{D}, \mathrm{N}=22 \mathrm{WT}, 19 \mathrm{Chrm}^{-1-}, 23 \mathrm{Chrml}^{-/}$and 12 dual $\mathrm{KO}$ mice. Size bars $=200$ micrometers.

\section{Genes differentially expressed in colon tumors from WT and $\mathrm{Chrm}^{-/-}$mice}

Tissues obtained in these experiments provided an opportunity to identify novel genes and signaling pathways potentially underlying tumor-promoting actions of M3R activation in the colon [6,11-14]. We designed an experimental strategy using gene microarray to compare gene expression profiles in tumors from $\mathrm{Chrm}^{-/-}$and WT mice, and thereby identify novel candidate M3R-regulated genes worthy of further exploration. To exclude changes in gene expression that resulted from neoplastic transformation but were not a specific consequence of changes in M3R expression, we used qPCR to validate key microarray findings and compare expression of candidate genes in tumors from $\mathrm{Chrm3}^{-/-}, \mathrm{Chrm1}^{-/}$, dual $\mathrm{KO}$ and WT mice. We reasoned that genes whose expression levels were different in tumors from $\mathrm{Chrm3}^{-/-}$compared to WT mice, but not in tumors from $\mathrm{Chrm1}^{-/-}$compared to WT mice, would more likely be relevant to M3R-induced colon tumor promotion. Although this approach has potential limitations we thought it would provide useful information regarding potential molecular pathways underlying the different tumor phenotypes.

We used an Illumina gene microarray to compare expression profiles for 19,100 genes in tumors from $\mathrm{Chrm}^{-/-}$ and WT mice. Using 474 probes, we identified 430 genes either up- or down-regulated by enrichment scores of at least 1.3 (equivalent to a non-log scale value of 0.05 ) in tumors from AOM-treated $\mathrm{Chrm}^{-/-}$mice compared to those from WT mice. Ingenuity Pathway Analysis of this data set did not reveal involvement of a known pathway to explain tumor attenuation in AOM-treated $\mathrm{Chrm}^{-/-}$compared to WT mice (data not shown).

Surmising that M3R-regulated genes important for colon tumor promotion would undergo relatively large changes in expression, for further analysis we arbitrarily set a cut-off at the top 14 down-regulated and top 14 up-regulated genes identified by microarray in tumors from $\mathrm{Chrm3}^{-/-}$compared to WT mice, a total of 28 genes (Figure 4A). The heat map deriving from this 'validation set' demonstrates consistent changes in gene expression in tumors from three mice from each genotype. There were large changes in expression of some genes. Fcerlg gene expression was nearly abolished in tumors from $\mathrm{Chrm3}^{-/}$compared to those from WT mice whereas Tmem14C gene expression was strongly up-regulated (Figure 4A), findings confirmed by qPCR using the same mRNA employed for the microarray analysis (not shown).

We used qPCR to examine expression of a subset of genes strongly associated with colon neoplasia [25]. Cyclin 


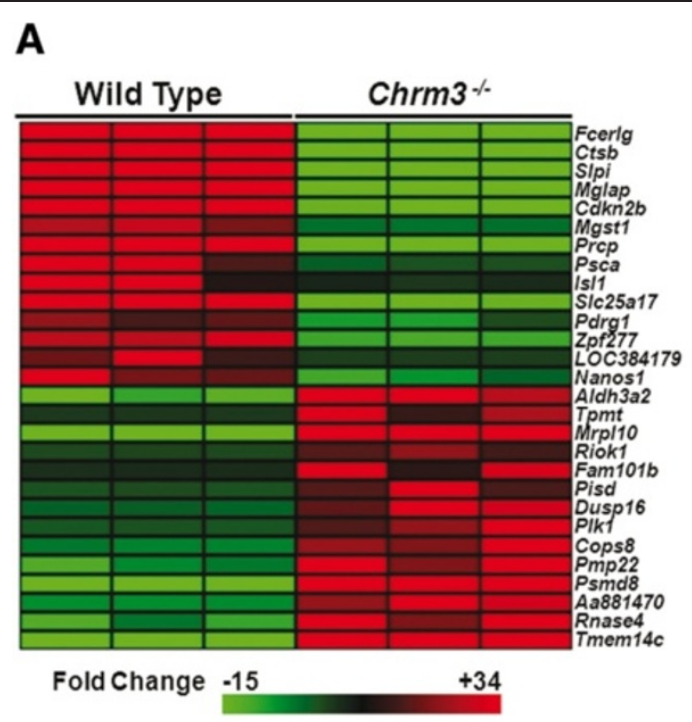

\begin{tabular}{|l|c|}
\hline Bouse Gene & $\begin{array}{c}\text { Gene Expression in } \\
\text { Tumors from Chrm3\%. } \\
\text { Compared to WT Mice } \\
\text { (fold change by qPCR) }\end{array}$ \\
\hline Fcer1g & 0.01 \\
\hline Psca & 0.02 \\
\hline Zfp277 & 0.17 \\
\hline Nanos1 & 0.19 \\
\hline Pmp22 & 0.24 \\
\hline Is/1 & 0.25 \\
\hline Prcp & 0.42 \\
Ctsb & 0.44 \\
Cops8 & 2.09 \\
\hline Fam101b & 2.35 \\
\hline Tmem14c & 2.53 \\
Dusp16 & 2.63 \\
Tpmt & 3.00 \\
Rnase4 & 4.70 \\
\hline
\end{tabular}

Figure 4 Analyses of gene expression in tumors from WT and $\mathrm{Chrm}^{-/-}$mice. A: Heat map displaying expression pattern of the 28 genes with the largest changes in expression when comparing tumors from Chrm $3^{-/-}$relative to tumors from WT mice. Rows represent genes and columns represent samples. Color intensity denotes the standardized ratio between each value and the average expression of each gene across all samples. Red pixels indicate increased abundance and green pixels decreased abundance of each transcript in the indicated sample. $\mathrm{N}=3$ mice/ genotype. B: Comparison of changes in murine gene expression levels in tumors from $\mathrm{Chrm}^{-1-}$ mice relative to tumors from WT mice quantified by real time RT-PCR (qPCR). WT, wild-type.

D1 expression was unchanged in tumors from $\mathrm{Chrm}^{-1-}$ mice compared to those from WT mice suggesting that changes in some genes key to intestinal neoplasia occurred prior to the 20-week end-point (Additional file 2). Nonetheless, at the 20-week time-point, expression of both $c$-myc and $\beta$-catenin in tumors from $\mathrm{Chrm}^{-/}$compared to those from WT mice was reduced $25 \%$, and Myd88 expression was reduced 32\% (Additional file 2). The greatest changes were in Egfr and Cox2 expression, reduced 54 and 70\%, respectively, in tumors from $\mathrm{Chrm}^{-/-}$ mice (Additional file 2). Altered Egfr expression was of particular interest since we showed previously that cross-talk between M3R and EGFR is critical for human colon cancer cell proliferation [6] and others showed that Egfr signaling in mice is required for AOM-induced colon neoplasia $[26,27]$. COX2 is also over-expressed in human colon neoplasia $[28,29]$ and, in mouse models of intestinal neoplasia, Cox2 (Ptgs2)-deficiency attenuates tumor formation [30]. We also confirmed the absence of compensatory increases in Chrm3 expression in $\mathrm{Chrm}^{-1-}$ mice that could account for the Chrm1 ${ }^{-/-}$colon tumor phenotype.

To validate changes in expression of the 28 genes shown in Figure 4A, we extracted additional mRNA from colon tumors from WT and $\mathrm{Chrm}^{-/}$mice and compared gene expression using qPCR. To more effectively separate signal from noise, for this analysis we selected an arbitrary conservative threshold of two-fold or greater changes in mRNA expression. Applying these more stringent criteria, we detected meaningful changes in the expression of mRNA for 14 genes derived from the original 28-gene set (Figure 4A); eight genes were down-regulated and six were up-regulated in tumors from $\mathrm{Chrm}^{-\%}$ compared to WT mice (Figure 4B). To our knowledge, none of the 14 genes in this 'core Chrm3-dependency gene set' were previously associated with muscarinic receptor signaling.

\section{Comparison of gene expression in colon tumors from $\mathrm{Chrm}^{-/-}, \mathrm{Chrm} 1^{--1}$, dual KO and WT mice}

To identify novel genes relevant to Chrm subtype-dependent differences in colon neoplasia, we used qPCR to measure expression of the core Chrm3-dependency gene set shown in Figure 4B using mRNA extracted from tumors from $\mathrm{Chrm3}^{--}, \mathrm{Chrm}^{-/-}$and dual $\mathrm{KO}$ mice, and then compared expression of these 14 genes to that in tumors from WT mice. Specifically, we sought genes whose expression levels in tumors from $\mathrm{Chrm}^{-/-}$and $\mathrm{Chrm1}^{-/-}$mice compared to those from WT mice were consistent with the different colon neoplasia phenotypes (Figure $2 \mathrm{~B}$ and $\mathrm{C}$ ); that is, we thought it important to pursue genes with meaningful changes in expression in tumors from $\mathrm{Chrm}^{-/}$compared to WT mice, but not in tumors from $\mathrm{ChrmI}^{-/}$compared to WT mice. Using this approach we identified five candidate mouse genes meeting these criteria - Tpmt, Prcp, Pmp22, Nanos1 and Zfp277. One gene (Tpmt) was up-regulated in tumors from $\mathrm{Chrm}^{-1}$ mice and four (Prcp, Pmp22, Nanos1 and Zfp277) were down- 
regulated (Figure 5A). However, the only change in gene expression that achieved statistical significance was reduced Zfp277 levels in tumors from $\mathrm{Chrm}^{-/-}$compared to those from WT and Chrm1 $1^{-/}$mice $(P<0.05$, Figure 5A).

Next, to determine whether similar changes in gene expression occurred in non-neoplastic colon, we used qPCR to compare expression levels of these five candidate genes in normal colon from WT, Chrm3 $3^{-/}, \mathrm{Chrm1}^{-/-}$and dual $\mathrm{KO}$ mice that had not been treated with either AOM or vehicle (Figure 5B). In these experiments, we used expression of an endogenous housekeeping gene, Gapdh, as an internal control; Gapdh expression did not change significantly in normal colon and tumors across mouse genotypes (not shown). Comparison of the data in Figure 5A and $\mathrm{B}$ revealed similar patterns of gene expression in tumors and normal colon tissue for Prcp, Pmp22, Nanos1 and $Z f p 277$, but not for Tpmt. Hence, we considered it likely that changes in expression of mRNA for Tpmt in tumors resulted from neoplastic transformation irrelevant to regulation by muscarinic receptor signaling; Tpmt was excluded from further consideration. Again, the only change in gene expression achieving statistical significance was reduced Zfp 277 levels in normal colon from $\mathrm{Chrm3}^{-/-}$ compared to expression in normal colon from WT and $\mathrm{Chrm}^{-/-}$mice $(P<0.05$, Figure $5 \mathrm{~B})$. Based on these collective findings, we selected $Z f p 277$ for further analysis and excluded Tpmt, Prcp, Pmp22 and Nanos1 from further consideration as genes relevant to M3R-regulated colon neoplasia.

\section{Zinc finger protein 277 expression in tumors from WT, M1R- and M3R-deficient mice}

To establish the functional relevance of changes in gene expression, we used immunohistochemistry with specific antibodies to interrogate colon tumor sections for changes in $\mathrm{Zfp} 277$ protein expression corresponding to changes in Zfp277 mRNA levels. In colon tumors from WT mice we observed robust Zfp277 staining (Figure 5C). Notably, Zfp277 protein expression was reduced approximately $50 \%$ in tumors from $\mathrm{Chrm3}^{-/-}$and dual $\mathrm{KO}$ mice compared to tumors from WT mice $(P<0.05)$ (Figure $5 \mathrm{C})$. As anticipated from the mRNA data, Zfp277 protein expression was the same in tumors from WT and $\mathrm{Chrm}^{-/-}$mice (Figure 5C). Taken together, these novel findings identify parallel changes in both gene and protein expression for Zfp277.

\section{Zinc finger protein 277 is over-expressed in human colon cancer}

As an initial exploration of the potential clinical significance of these findings, we searched human expression data sets in the National Center for Biotechnology
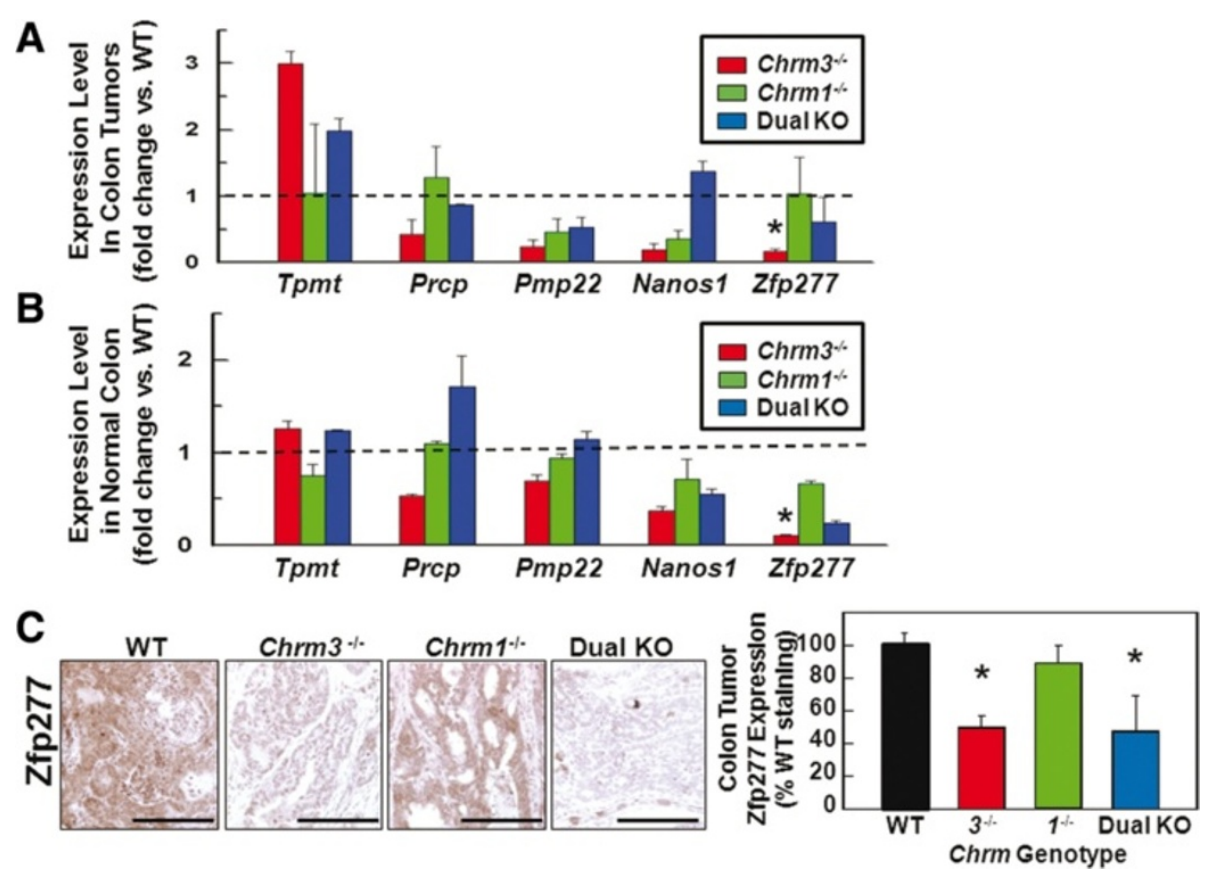

Figure 5 Expression levels of murine genes quantified by qPCR in tumor and normal colon tissue, and Zfp277 protein expression in tumor sections from WT, Chrm3 $3^{-/}, \mathrm{Chrm} \mathrm{1}^{--}$and dual KO mice. A: Colon tumors. N=3 mice/genotype. B: Normal colon. $\mathrm{N}=3$ mice/genotype. Shown is the fold difference in gene expression normalized to glyceraldehyde-3-phosphate dehydrogenase (Gapdh) levels in tissue from Chrm $3^{-/}$, $\mathrm{Chrm}^{-\%}$ and dual $\mathrm{KO}$ mice relative to that in tissue from WT mice. Error bars, S.E. (* indicates $\left.P<0.05\right)$. C: Representative images show immunohistochemical staining with anti-Zfp277 antibody on tumor sections from WT, Chrm ${ }^{-1}, \mathrm{Chrm}^{-1}$ and dual $\mathrm{KO}$ mice. Graph shows quantification of Zfp277 staining in tumor sections. Data shown represent mean \pm S.E. from four mice per genotype ${ }^{*}$ indicates $\left.P<0.05\right)$. Size bars $=100$ micrometers. 
Information (NCBI) Gene Expression Omnibus (GEO) Profiles database. Specifically, in datasets of human colon cancer compared to adjacent normal colon tissue from the same person we sought information regarding the expression of ZNF277, the human analogue of $Z f p 277$. This database search yielded only one relevant study of surgical specimens comparing gene expression profiles in colon cancer compared to normal colon [31]. Our in silico analysis of this existing dataset revealed that ZNF277 expression was significantly up-regulated in 33 of 34 colon cancers compared to adjacent normal colon $\left(P=2 \times 10^{-8}\right)[31]$.

We sought to confirm this observation in our lab by interrogating a set of 12 archived colon cancer tissues with adjacent normal colon that had been flash-frozen immediately after surgery at our own institution and stored at $-80^{\circ} \mathrm{C}$. In Figure $6 \mathrm{~A}$, changes in mRNA expression in colon cancer compared to normal colon are depicted in order of increasing CHRM3 expression (black bars). Although increases in ZNF277 mRNA were not as robust as those for CHRM3 mRNA, the expression patterns were very similar (Figure 6A). In these 12 human tissue samples, over-expression of ZNF277 in colon cancer was contingent upon over-expression of CHRM3 (Spearman rank correlation coefficient $=0.73$ ).

Next, we validated the importance of these changes in gene expression by measuring and comparing M3R and ZNF277 protein expression in an archived set of 23 formalin-fixed paraffin-embedded colon cancer tissues with adjacent normal colon from the same patients. We analyzed these samples immunohistochemically using specific antibodies for M3R and ZNF277 (Figure 6B). As shown in Figure 6C, zinc finger protein 277 and M3R were both significantly over-expressed in cancer compared to adjacent normal tissue. Moreover, as we observed for gene expression, statistical analysis of these

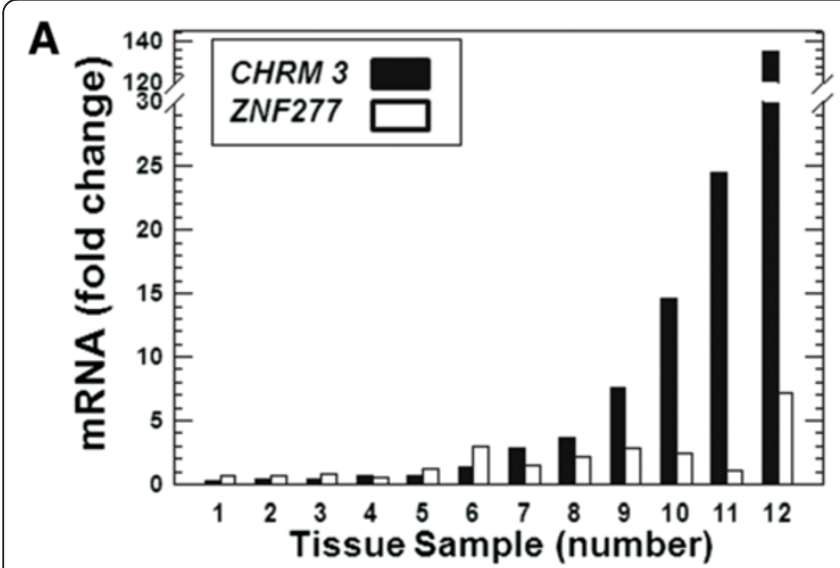

B

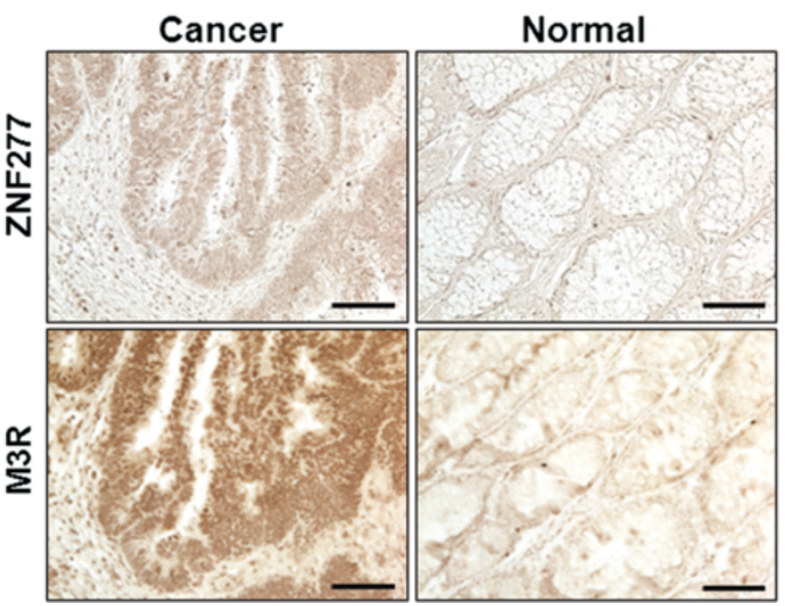

C

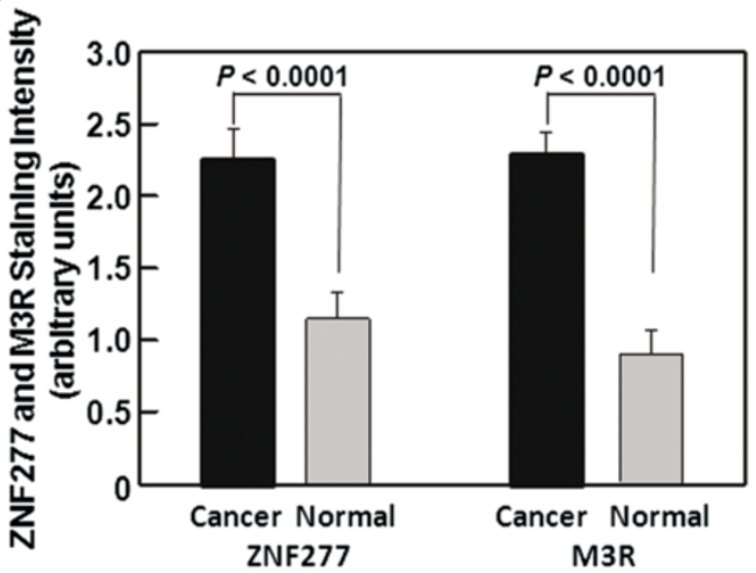

Figure 6 M3R and ZNF277 gene and protein expression in human colon cancer compared to adjacent normal colon. A: CHRM3 and ZNF277 mRNA levels in 12 sets of colon cancer and adjacent normal colon obtained from the same patients. mRNA levels were first normalized to expression of $\beta 2$-microglobulin and then ranked by increasing levels of CHRM3 expression. B: Representative images show immunohistochemical staining with anti-ZNF277 and anti-M3R antibodies on human colon cancer and adjacent normal colon epithelial tissue. Size bars $=100$ micrometers. C: Graph shows quantification of ZNF277 and M3R staining in cancer compared to normal colon. Data shown represent mean \pm S.E. of 23 sets of colon cancer and adjacent normal colon tissues. 
human tissue data indicated that over-expression of ZNF277 and M3R protein were also contingent variables; i.e., ZNF277 over-expression was statistically associated with M3R over-expression $(P=0.04)$. Collectively, these data support the novel conclusion that in both murine and human colon cancer, zinc finger protein 277 plays a currently undefined role in M3R-mediated promotion of neoplasia.

\section{Discussion}

Experimental mouse models that mimic clinical and molecular aspects of human colon cancer progression are useful tools to identify novel mechanisms underlying colon neoplasia [24]. Here, to explore the individual roles and interplay between two muscarinic receptor subtypes, we analyzed systematically the effects of Chrm3 and Chrm1 gene ablation, alone and in combination, on AOM-induced murine colon neoplasia, a model closely resembling non-hereditary human colon cancer [24]. To gain additional genetic and potentially mechanistic insights, we used a hypothesis-unbiased approach to elucidate concomitant changes in gene expression levels in tumors from WT and M3R-deficient mice.

As anticipated from our previous work [19,20], tumor number and size as well as tumor cell proliferation measured by Ki67 staining were greatly attenuated in AOMtreated $\mathrm{Chrm3}^{-/}$compared to WT mice. Based on our previous findings regarding the interplay of M1R and M3R in gastric function [21], we hypothesized that compared to the effects of Chrm3 gene ablation alone, concurrent ablation of Chrm3 and Chrm1 might further attenuate, or even abolish, AOM-induced tumor formation. Instead, we were surprised to observe that Chrm1 gene ablation alone did not alter tumor formation. We were further surprised to observe that combined Chrm3 and Chrm1 knockout mitigated reductions in tumor number and size observed with Chrm3 knockout alone.

We can draw several novel conclusions from these observations. Whereas work from our [19] and other groups $[2-4,7,8]$ provide strong evidence that M3R acts as a tumor promoter, the present observations newly suggest that in the colon M1R acts as a tumor suppressor. This putative role for M1R is unmasked by combined M1R and M3R deficiency in dual $\mathrm{KO}$ mice where tumor formation is similar to that observed in WT mice. Thus, M1R deficiency appears to negate the beneficial effects of knocking out only M3R. This finding may also explain why treating $A p c^{\text {min/+ }}$ mice with a non-selective muscarinic receptor inhibitor, scopolamine butylbromide, attenuates intestinal tumor formation less efficaciously than M3R gene ablation [20]. Scopolamine butylbromide treatment blocks both M3R and M1R activation, thereby mimicking combined M3R and M1R deficiency in dual KO mice. These considerations intimate therapeutic promise for a pharmacological approach to block M3R activation while at the same time augmenting M1R activation.

The unanticipated findings regarding the impact of Chrm3 and Chrm1 knockout on AOM-induced colon neoplasia provided an opportunity to explore M3R-regulated changes in tumor gene expression. We identified a set of genes expressed differentially in tumors from WT and M3R-deficient mice. Then, we used tumors from M1R-deficient mice as controls to exclude non-specific changes in gene expression due to neoplastic transformation but not a specific consequence of altered M3R expression. We employed a gene microarray comprising 19,100 target genes to identify 430 genes with expression levels significantly altered in tumors from M3Rdeficient compared to WT mice (strategy schematized in Additional file 3); a dataset further refined by increasing statistical stringency and using qPCR to validate results. These combined approaches identified 14 promising genes with meaningful changes in expression in tumors from M3R-deficient compared to WT mice (Figure 4B).

We concede this gene discovery approach has limitations. It may have identified only a fraction of the genome; many genes are turned off or encode proteins required for survival in specific amounts that do not change. Also, protein expression may be regulated by mechanisms that do not involve altered mRNA levels. Financial constraints limited expression profiling experiments to a relatively small number of observations under identical conditions and for the same reason limited further investigation of candidate genes to the relatively small subset of 14 genes shown in Figure 4B, thereby reducing statistical power. We may have missed important but subtle changes in gene expression. Even so, we believe that confirmatory results from our qPCR experiments provided reliable measures of changes in the expression levels of this 14gene subset in both tumors and normal colon from WT, $\mathrm{Chrm3}^{-/-}, \mathrm{Chrm}^{-/-}$and dual $\mathrm{KO}$ mice.

Changes in expression of only one gene, Zfp277, achieved statistical significance with matching changes in expression of the corresponding protein. Based on the stringent overall approach (Additional file 3), we are confident that our work identifies a novel role for $Z f p 277$ as an M3R-regulated gene pertinent to the progression of intestinal neoplasia. Confidence in this conclusion was bolstered by detecting over-expression of both mRNA and protein for ZNF277, the human analogue of Zfp277, in human colon cancer samples and, importantly, that this over-expression mirrored that of CHRM3 and M3R.

Literature and gene bank searches revealed little regarding the function of ZNF277 (NIRF4), which is expressed in multiple tissues, including the proximal colon [32]. The protein product, zinc finger protein 277 , is reported to play a role in cellular senescence and protection against genomic instability and cancer [33]. ZNF277 over-expression 
is reported in other cancers - chronic lymphocytic leukemia, well-differentiated renal cell carcinoma, and germ cell and endocrine tumors [32]. Hence, a novel, hitherto unrecognized role for ZNF277 in colon cancer biology is certainly plausible. We found no previous reports of an association between either $Z f p 277$ or ZNF277 expression and muscarinic receptor expression or activation. In future work, we plan to use in vitro and in vivo models to explore the molecular mechanisms underlying the association between M3R and ZNF277 expression.

\section{Conclusions}

Our results identify a novel candidate mouse gene, Zfp277, whose expression pattern is compatible with a role in mediating divergent effects of Chrm3 and Chrm1 gene ablation on murine intestinal neoplasia. Although finding an association between ZNF277 and M3R overexpression in human colon cancer is reassuring, we do not currently understand the molecular mechanism underlying this interaction. Future work will use both in vitro and in vivo approaches to address these questions and elucidate the functional role of ZNF277 and its interaction with $\mathrm{M} 3 \mathrm{R}$ in colon cancer. Although it is currently unclear why M3R and M1R, which both signal by stimulating phospholipid turnover and changes in cell calcium [10], have such divergent effects on colon neoplasia, their contrary roles suggest that jointly targeting these receptors may have therapeutic potential. Based on these considerations, we are optimistic that improved understanding of the role of muscarinic receptors in neoplasia will continue to yield novel therapeutic targets for colon cancer.

\section{Methods}

Animals

$\mathrm{Chrm}^{-/-}$and $\mathrm{Chrm1}^{-/-}$mice were generated from the same mixed genetic background (129S6/SvEvTac X CF1: 50\%/ $50 \%)$ as described previously [23,34,35]. Dual KO mice on the same genetic background were generated by mating homozygous $\mathrm{Chrm1}^{-/-}$and $\mathrm{Chrm}^{-/-}$mutant mice [36]. For all experiments, only male mice were used and agedmatched WT mice of the same genetic background served as controls. Mice were housed under identical conditions in a pathogen-free room, had free access to commercial rodent chow and water, and were acclimatized in the vivarium for at least one week before experiments. These studies were approved by the University of Maryland School of Medicine Institutional Animal Care and Use, and the Baltimore VA Research and Development Committees.

\section{Human tissues}

To examine M3R (CHRM3) and ZNF277 gene and protein expression, we used archived pre-existing de-identified surgical specimens of colon cancer and adjacent normal colon epithelium (approved by the University of Maryland School of Medicine Institutional Review Board and the Baltimore VA Research and Development Committee).

\section{Study design}

For the initial 6 weeks of treatment, 94 mice (25 WT, 20 $\mathrm{Chrm3}^{-/}, 23 \mathrm{Chrm1}^{-/-}$and 26 dual $\mathrm{KO}$ mice) received weekly intraperitoneal injections of azoxymethane (AOM; Midwest Research Institute; $10 \mathrm{mg} / \mathrm{kg}$ body weight) and 21 mice (5 WT, $5 \mathrm{Chrm}^{-/-}, 5 \mathrm{Chrml}^{-/-}$and 6 dual $\mathrm{KO}$ mice) received an equal volume of vehicle (phosphatebuffered saline) (Figure 1A). As in our previous study [19], AOM and vehicle treatment in WT, $\mathrm{Chrm}^{-/-}$and $\mathrm{Chrm}^{-/-}$mice was started when animals were 6-weeks old. In dual $\mathrm{KO}$ mice, AOM and PBS treatments were initiated at 12 weeks of age. All animals were euthanized 20 weeks after initiating AOM injections. Colon length was measured, and segments were opened longitudinally and placed flat on microscope slides. Tumors were identified by visual inspection and photographed (Nikon SMZ1500 dissecting microscope). Tumor size was measured using calipers and tumor volume calculated using: volume $=1 / 2\left(\right.$ length $\times$ width $\left.^{2}\right)$ [37].

\section{Histological and immunohistochemical staining analysis}

Tissues were fixed in $4 \%$ paraformaldehyde and paraffinembedded. Five-micrometer sections were stained with hematoxylin and eosin. Adenomas and adenocarcinomas were defined according to consensus recommendations by the Mouse Models of Human Cancers Consortium [38]. As markers of cell proliferation and apoptosis, we used immunohistochemical staining for Ki67 and activated caspase-3, respectively (antibodies from Cell Signaling Technology). Only complete crypts were evaluated and investigators were masked to genotype and treatment group.

To identify corresponding changes in protein expression for relevant genes identified by microarray and qPCR, formalin-fixed paraffin-embedded tumor sections were immunostained with a specific antibody against both mouse Zfp277 and human ZNF277 from Santa Cruz Biotech (Santa Cruz, CA), and a specific antibody from Alomone Labs (Jerusalem, Israel) against both mouse and human M3R. Tumor sections were examined with a Nikon $80 i$ photomicroscope at $200 \times$ magnification. Sections were first reviewed and scored by a senior pathologist (CD) masked to tissue origin and immunostaining was then quantified using Image-Pro Plus software (version 5.1; Media Cybernetics, Silver Spring, MD). To minimize variation, all tumor sections were examined and photographed using the same microscope settings. 


\section{Microarray performance and analyses}

After resection, murine tissue was immediately stored in RNAlater (Ambion) at $-80^{\circ} \mathrm{C}$. Total RNA was extracted using the RNeasy kit from Qiagen. RNA was digested using the RNase-Free DNase set. The quality of total RNA was tested and confirmed using a Bioanalyzer 2100 (Expression Analysis, Inc., Durham, NC). The microarray assay was performed by Expression Analysis, Inc. using the MouseWG-6 v2.2 Expression BeadChip (Illumina, San Diego, CA). This chip covers the whole mouse genome, > 19,100 unique, curate genes targeting a total of 45,281 transcripts. Results were analyzed using the cubic spline normalization method without background subtraction $[39,40]$. In comparing changes in mRNA expression in $\mathrm{Chrm3}^{-/-}$vs. WT mouse colon tumors, statistical significance cutoff levels were set for individual transcripts at $P \leq 0.05$ (false discovery rate) and enrichment scores $\geq \pm$ 1.3; changes in gene mRNA that met these thresholds were deemed to be differentially expressed. Microarray data represent results of tissue from three different mice per genotype. Results were submitted to the National Center for Biotechnology Information (NCBI) Gene Expression Omnibus (GEO) database (GSE43444).

\section{Quantitative RT-PCR (qPCR)}

First-strand cDNAs were synthesized from $5 \mu \mathrm{g}$ RNA (Superscript III First Strand Synthesis System for RTPCR, Invitrogen). qPCR was then performed using $50 \mathrm{ng}$ cDNA, the SYBR Green PCR Master Mix (Applied Biosystems), and forward and reverse primers (final concentration $0.5 \mu \mathrm{M}$ in sample volumes of $20 \mu \mathrm{l}$ ). Primers (Additional file 4) were designed to span introns using the National Center for Biotechnology Information nucleotide database SIM-4 gene alignment program and on-line software (http://www.genscript.com/index.html). qPCR was performed using the 7900HT Fast System (ABI) with Power SYBR Green Master Mix (ABI). PCR conditions included $5 \mathrm{~min}$ at $95^{\circ} \mathrm{C}$ followed by 37 cycles of $95^{\circ} \mathrm{C}$ for 15 seconds, $60^{\circ} \mathrm{C}$ for 20 seconds, and $72^{\circ} \mathrm{C}$ for 40 seconds and a final cycle at $95^{\circ} \mathrm{C}$ for 15 seconds, $60^{\circ} \mathrm{C}$ for 15 seconds, and $95^{\circ} \mathrm{C}$ for 15 seconds. PCR data were analyzed using ABI instrument software SDS 2.1. Expression of candidate genes in each group of mice was normalized to glyceraldehyde 3-phosphate dehydrogenase (Gapdh). For human samples, expression of CHRM3 and ZNF277 was normalized to $\beta_{2}$-microglobulin $(B 2 M)$, a preferable housekeeping gene for analysis of colon cancer [41]. Quantitative qPCR data were evaluated using the comparative $C_{\mathrm{T}}\left(2^{-\Delta \Delta C \mathrm{~T}}\right)$ method [42].

\section{Statistical analysis}

Student's unpaired $t$-test was used to determine statistical significance. The strength of linear association between two variables was quantified using the Spearman's rank correlation coefficient and that of non-linear associations by Pearson's chi-squared test. $P$ values $\leq 0.05$ were considered significant.

\section{Additional files}

Additional file 1: Delaying initiation of AOM treatment until mice are 12-weeks-old rather than 6-weeks-old does not alter colon tumor number. Comparison of tumor number (left panel) and tumor volume (right panel) 20 weeks after starting AOM treatment in 6- and 12-week-old WT mice (mean \pm S.E.). $N$ represents number of mice per treatment group

Additional file 2: Comparison of changes in expression of key genes associated with colon neoplasia in tumors from $\mathrm{Chrm}^{-1-}$ mice relative to tumors from WT mice quantified by real time RT-PCR (qPCR).

Additional file 3: Experimental strategy used to identify changes in gene and protein expression relevant to divergent effects of muscarinic receptor gene ablation on colon neoplasia phenotypes. The experimental approaches, comparators and yields (outcomes) for each stage of investigation are outlined. An enrichment score of 1.3 is equivalent to a non-log scale value of 0.05 . qPCR, quantitative RT-PCR; WT, wild-type; IHC, immunohistochemical analysis.

Additional file 4: RT-PCR (qPCR) primers used in this study.

\section{Competing interests}

The authors declare that they have no competing interests.

\section{Authors' contributions}

KC, GX, SK and JPR conceived the study design and designed the experiments. KC, SK, JT, NS and JPR carried out the study. JPR prepared the manuscript. KC, SK and JPR supervised the statistical analysis. KC, GX, SK, $C D$ and JPR revised the manuscript. $K C, J H$ and $C D$ performed the histopathological assessments of animal and human tissue. KC, GX, SK and JPR supervised the interpretations of results and revised the manuscript. All authors read and approved the final manuscript.

\section{Acknowledgements}

We thank Dr. Jürgen Wess, Molecular Signaling Section, Laboratory of Bioorganic Chemistry, National Institute of Diabetes and Digestive and Kidney Diseases, National Institutes of Health, Bethesda, MD, for kindly providing knockout mice used in this work and for helpful suggestions and insights during the course of this work. We thank Xue-Min Gao, University of Maryland School of Medicine, for technical assistance.

This work was supported by the Office of Research and Development, Medical Research Service, Department of Veterans Affairs (to J-P. Raufman) and by National Institutes of Health grant numbers R01 CA107345, R01 CA120407, R21 DK093406 (to J-P. Raufman), K08 DK080843 (to G. Xie) and K08 DK081479 (to S. Khurana). J. Timmons and N. Shah were supported by National Institutes of Health grant number T32 DK067872 (to J-P. Raufman)

\section{Author details}

${ }^{1}$ Department of Medicine, Division of Gastroenterology \& Hepatology and Program in Oncology, Marlene and Stewart Greenebaum Cancer Center, VA Maryland Health Care System and University of Maryland School of Medicine, Baltimore, MD 21201-1595, USA. ²Department of Medicine, Division of Gastroenterology, Georgia Regents University, Augusta, GA 30912, USA. ${ }^{3}$ Department of Pathology, Division of Anatomic Pathology, University of Maryland School of Medicine, Baltimore, MD 21201-1595, USA. ${ }^{4}$ Department of Surgery, University of Maryland School of Medicine, Baltimore, MD 21201-1595, USA. ${ }^{5}$ Division of Gastroenterology \& Hepatology, 22 S. Greene St., N3W62, Baltimore, MD 21201-1595, USA.

Received: 7 January 2014 Accepted: 27 March 2014 Published: 3 April 2014 


\section{References}

1. Song P, Sekhon HS, Jia Y, Keller JA, Blusztajn JK, Mark GP, Spindel ER: Acetylcholine is synthesized by and acts as an autocrine growth factor for small cell lung carcinoma. Cancer Res 2003, 63:214-221.

2. Song P, Sekhon HS, Lu A, Arredondo J, Sauer D, Gravett C, Mark GP, Grando SA, Spindel ER: M3 muscarinic receptor antagonists inhibit small cell lung carcinoma growth and mitogen-activated protein kinase phosphorylation induced by acetylcholine secretion. Cancer Res 2007, 67:3936-3944

3. Jimenez E, Montiel M: Activation of MAP kinase by muscarinic cholinergic receptors induces cell proliferation and protein synthesis in human breast cancer cells. J Cell Physiol 2005, 204:678-686.

4. Rimmaudo LE, de la Torre E, Sacerdote de Lustig E, Sales ME: Muscarinic receptors are involved in LMM3 tumor cells proliferation and angiogenesis. Biochem Biophys Res Commun 2005, 334:1359-1364.

5. Rayford W, Noble MJ, Austenfeld MA, Weigel J, Mebust WK, Shah GV: Muscarinic cholinergic receptors promote growth of human prostate cancer cells. Prostate 1997, 30:160-166.

6. Cheng K, Zimniak P, Raufman JP: Transactivation of the epidermal growth factor receptor mediates cholinergic agonist-induced proliferation of H508 human colon cancer cells. Cancer Res 2003, 63:6744-6750.

7. Ukegawa Jl, Takeuchi Y, Kusayanagi S, Mitamura K: Growth-promoting effect of muscarinic acetylcholine receptors in colon cancer cells. $J$ Cancer Res Clin Oncol 2003, 129:272-278.

8. Boss A, Oppitz M, Lippert G, Drews U: Muscarinic cholinergic receptors in the human melanoma cell line SK-Mel 28: modulation of chemotaxis. Clin Exp Dermatol 2005, 30:557-564.

9. Shah N, Khurana S, Cheng K, Raufman JP: Muscarinic receptors and ligands in cancer. Am J Physiol Cell Physiol 2009, 296:C221-C232.

10. Wess J: Molecular biology of muscarinic acetylcholine receptors. Crit Rev Neurobiol 1996, 10:69-99.

11. Cheng K, Samimi R, Xie G, Shant J, Drachenberg C, Wade M, Davis RJ, Nomikos G, Raufman JP: Acetylcholine release by human colon cancer cells mediates autocrine stimulation of cell proliferation. Am J Physiol Gastrointest Liver Physiol 2008, 295:G591-G597.

12. Shant J, Cheng K, Marasa BS, Wang JY, Raufman JP: Akt-dependent NF-kappaB activation is required for bile acids to rescue colon cancer cells from stress-induced apoptosis. Exp Cell Res 2009, 315:432-450.

13. Belo A, Cheng K, Chahdi A, Shant J, Xie G, Khurana S, Raufman JP: Muscarinic receptor agonists stimulate human colon cancer cell migration and invasion. Am J Physiol Gastrointest Liver Physiol 2011, 300:G749-G760.

14. Raufman JP, Cheng K, Saxena N, Chahdi A, Belo A, Khurana S, Xie G: Muscarinic receptor agonists stimulate matrix metalloproteinase 1dependent invasion of human colon cancer cells. Biochem Biophys Res Commun 2011, 415:319-324

15. Hanahan D, Weinberg RA: Hallmarks of cancer: the next generation. Cell 2011, 144:646-674

16. Wess J: Muscarinic acetylcholine receptor knockout mice: novel phenotypes and clinical implications. Annu Rev Pharmacol Toxicol 2004, 44:423-450

17. Wess J, Eglen RM, Gautam D: Muscarinic acetylcholine receptors: mutant mice provide new insights for drug development. Nat Rev Drug Discov 2007, 6:721-733

18. Wess J: Novel muscarinic receptor mutant mouse models. Handb Exp Pharmacol 2012, 208:95-117.

19. Raufman JP, Samimi R, Shah N, Khurana S, Shant J, Drachenberg C, Xie G, Wess J, Cheng K: Genetic ablation of M3 muscarinic receptors attenuates murine colon epithelial cell proliferation and neoplasia. Cancer Res 2008, 68:3573-3578

20. Raufman JP, Shant J, Xie G, Cheng K, Gao XM, Shiu B, Shah N, Drachenberg CB, Heath J, Wess J, Khurana S: Muscarinic receptor subtype-3 gene ablation and scopolamine butylbromide treatment attenuate small intestinal neoplasia in Apcmin/+ mice. Carcinogenesis 2011, 32:1396-1402.

21. Xie G, Drachenberg C, Yamada M, Wess J, Raufman JP: Cholinergic agonistinduced pepsinogen secretion from murine gastric chief cells is mediated by M1 and M3 muscarinic receptors. Am J Physiol Gastrointest Liver Physiol 2005, 289:G521-G529.

22. Rosemond E, Rossi M, McMillin SM, Scarselli M, Donaldson JG, Wess J: Regulation of $\mathrm{M}$ muscarinic receptor expression and function by transmembrane protein 147. Mol Pharmacol 2011, 79:251-261.
23. Yamada M, Miyakawa T, Duttaroy A, Yamanaka A, Moriguchi T, Makita R, Ogawa M, Chou CJ, Xia B, Crawley JN, Felder CC, Deng CX, Wess J: Mice lacking the M3 muscarinic acetylcholine receptor are hypophagic and lean. Nature 2001, 410:207-212.

24. Neufert C, Becker C, Neurath MF: An inducible mouse model of colon carcinogenesis for the analysis of sporadic and inflammation-driven tumor progression. Nat Protoc 2007, 2:1998-2004.

25. Markowitz SD, Bertagnolli MM: Molecular origins of cancer: molecular basis of colorectal cancer. N Engl J Med 2009, 361:2449-2460.

26. Fichera A, Little N, Jagadeeswaran S, Dougherty U, Sehdev A, Mustafi R, Cerda S, Yuan W, Khare S, Tretiakova M, Gong C, Tallerico M, Cohen G, Joseph L, Hart J, Turner JR, Bissonnette M: Epidermal growth factor receptor signaling is required for microadenoma formation in the mouse azoxymethane model of colonic carcinogenesis. Cancer Res 2007, 67:827-835.

27. Dougherty U, Cerasi D, Taylor I, Kocherginsky M, Tekin U, Badal S, Aluri L, Sehdev A, Cerda S, Mustafi R, Delgado J, Joseph L, Zhu H, Hart J, Threadgill $D$, Fichera A, Bissonnette M: Epidermal growth factor receptor is required for colonic tumor promotion by dietary fat in the azoxymethane/dextran sulfate sodium model: roles of transforming growth factor-\{alpha\} and PTGS2. Clin Cancer Res 2009, 15:6780-6789.

28. Eberhart CE, Coffey RJ, Radhika A, Giardiello FM, Ferrenbach S, DuBois RN: Up-regulation of cyclooxygenase 2 gene expression in human colorectal adenomas and adenocarcinomas. Gastroenterology 1994, 107:1183-1188.

29. Sinicrope FA, Lemoine $M, X i$, Lynch PM, Cleary KR, Shen Y, Frazier ML: Reduced expression of cyclooxygenase 2 proteins in hereditary nonpolyposis colorectal cancers relative to sporadic cancers. Gastroenterology 1999, 117:350-358

30. Oshima M, Dinchuk JE, Kargman SL, Oshima H, Hancock B, Kwong E, Trzaskos JM, Evans JF, Taketo MM: Suppression of intestinal polyposis in Apc delta716 knockout mice by inhibition of cyclooxygenase 2 (COX-2) Cell 1996, 87:803-809.

31. Khamas A, Ishikawa T, Shimokawa K, Mogushi K, lida S, Ishiguro M Mizushima H, Tanaka H, Uetake H, Sugihara K: Screening for epigenetically masked genes in colorectal cancer Using 5-Aza-2'-deoxycytidine, microarray and gene expression profile. Cancer Genomics Proteomics 2012, 9:67-75

32. Liang H, Guo W, Nagarajan L: Chromosomal mapping and genomic organization of an evolutionarily conserved zinc finger gene ZNF277. Genomics 2000, 66:226-228.

33. Negishi M, Saraya A, Mochizuki S, Helin K, Koseki H, Iwama A: A novel zinc finger protein Zfp277 mediates transcriptional repression of the Ink4a/ arf locus through polycomb repressive complex 1. PLoS One 2010, 5:e12373.

34. Fisahn A, Yamada M, Duttaroy A, Gan JW, Deng CX, McBain CJ, Wess Muscarinic induction of hippocampal gamma oscillations requires coupling of the M1 receptor to two mixed cation currents. Neuron 2002, 33:615-624

35. Miyakawa T, Yamada M, Duttaroy A, Wess J: Hyperactivity and intact hippocampus-dependent learning in mice lacking the M1 muscarinic acetylcholine receptor. J Neurosci 2001, 21:5239-5250.

36. Gautam D, Heard TS, Cui Y, Miller G, Bloodworth L, Wess J: Cholinergic stimulation of salivary secretion studied with M1 and M3 muscarinic receptor single- and double-knockout mice. Mol Pharmacol 2004, 66:260-267.

37. Inaba M, Kobayashi T, Tashiro T, Sakurai Y, Maruo K, Ohnishi Y, Ueyama Y, Nomura T: Evaluation of antitumor activity in a human breast tumor/ nude mouse model with a special emphasis on treatment dose. Cancer 1989, 64:1577-1582.

38. Boivin GP, Washington K, Yang K, Ward JM, Pretlow TP, Russell R, Besselsen DG, Godfrey VL, Doetschman T, Dove WF, Pitot HC, Halberg RB, Itzkowitz $\mathrm{SH}$, Groden J, Coffey RJ: Pathology of mouse models of intestinal cancer: consensus report and recommendations. Gastroenterology 2003, 124:762-777.

39. Workman C, Jensen $L$, Jarmer $H$, Berka R, Gautier L, Nielser HB, Saxild HH, Nielsen C, Brunak S, Knudsen S: A new non-linear normalization method for reducing variability in DNA microarray experiments. Genome Biol 2002, 3:research0048.

40. Schmid R, Baum P, Ittrich C, Fundel-Clemens K, Huber W, Brors B, Eils R, Weith A, Mennerich D, Quast K: Comparison of normalization methods for Illumina BeadChip HumanHT-12 v3. BMC Genomics 2010, 11:349. 
41. Dydensborg AB, Herring E, Auclair J, Tremblay E, Beaulieu JF: Normalizing genes for quantitative RT-PCR in differentiating human intestinal epithelial cells and adenocarcinomas of the colon. Am J Physiol Gastrointest Liver Physiol 2006, 290:G1067-G1074.

42. Schmittgen TD, Livak KJ: Analyzing real-time PCR data by the comparative C(T) method. Nat Protoc 2008, 3:1101-1108.

doi:10.1186/1476-4598-13-77

Cite this article as: Cheng et al:: Divergent effects of muscarinic receptor subtype gene ablation on murine colon tumorigenesis reveals

association of M3R and zinc finger protein 277 expression in colon neoplasia. Molecular Cancer 2014 13:77.

\section{Submit your next manuscript to BioMed Central and take full advantage of:}

- Convenient online submission

- Thorough peer review

- No space constraints or color figure charges

- Immediate publication on acceptance

- Inclusion in PubMed, CAS, Scopus and Google Scholar

- Research which is freely available for redistribution 